Artículo de Reflexión E11A13. * Recibido 12-08-2017. * Aprobado versión final 15-05-2018. * Clasificación * JEL: M14. * Pp: 179-196 * doi: 10.33571/teuken.v12n18a9

\title{
Calidad del turismo sostenible desde la perspectiva del cliente.
}

\author{
Quality of sustainable tourism \\ from the customer perspective.
}

\author{
María Macarena Suárez Blanco \\ URUGUAY
}

Resumen: debido a la importancia económica que tiene en Uruguay, el turismo debería ser una actividad sostenible para la rentabilidad de las empresas, así como para la ecología y la cultura; incluso, desde la óptica de la economía verde, debería serlo también para la sociedad en general y la comunidad. Hoy, el turista exige propuestas de calidad; lo que supone que además de brindar el servicio de alojamiento, gastronomía y entretenimientos, el turismo debe considerar todos los factores antes mencionados, sin lo que no se lograría la plena satisfacción del servicio. La toma de decisiones del cliente será mejor en la medida en que se jerarquicen las alternativas.

Palabras claves: turismo sostenible, calidad del turismo, desarrollo del turismo, atención selectiva en turismo.

Abstract: Due to its economic importance in Uruguay, tourism should be a sustainable activity for the profitability of companies and ecology and culture; even from the perspective of the green economy, it should also be so for society in general and the community. Today, the tourist demands quality proposals, which means that in addition to providing accommodation, gastronomy, and entertainment services, tourism must consider all the factors above, without which full-service satisfaction would not be achieved. The client's decisionmaking will be better to the extent that the alternatives are ranked.

Keywords: Sustainable tourism, tourism quality, tourism development, tourism selective attention.

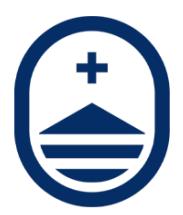

María Macarena Suárez es Contadora Pública de la Universidad Católica del Uruguay Dámaso Antonio Larrañaga; postgraduada en Comunicación Organizacional (UCUDAL) y estudiante de Maestría en Estudios Organizacionales en la UCUDAL. Es consultora y tallerista en inclusión financiera, Integrante de la cátedra de Economía UCUDAL y del Grupo de investigación Gestión de intangibles, turismo, comunicación organizacional, (UCUDAL).

Contacto: masuabla@icloud.com 


\section{Qualidade do turismo sustentável do ponto de vista do cliente}

Resumo: por sua importância econômica no Uruguai, o turismo deve ser uma atividade sustentável para a rentabilidade das empresas, ecologia e cultura; até, do ponto de vista da economia verde, deveria sê-lo também para a sociedade em geral e para a comunidade. Hoje, o turista exige propostas de qualidade; o que significa que para além da prestação de serviços de alojamento, gastronomia e animação, o turismo deve ter em consideração todos os factores acima mencionados, sem os quais não se alcançaria a plena satisfação com o serviço. A tomada de decisão do cliente será melhor na medida em que as alternativas forem classificadas.

Palavras-chave: turismo sustentável, turismo qualidade, turismo desenvolvimento, atenção seletiva do turismo.

\section{Introducción}

a Organización Mundial del Turismo (OMT) define al turismo, como las actividades y procedimientos que llevan a cabo las personas, en un lapso de tiempo inferior a un año, para realizar viajes a destinos diferentes al lugar en que residen. Los objetivos de estos viajes son muy variados; pueden ser por: placer, ocio, negocios o estudios, entre otros tantos motivos. En el país receptor se conforma un conjunto de fenómenos en respuesta al ingreso de las personas en ese lapso de tiempo. La propia OMT precisa que este desplazamiento es fuera del área geográfica donde la persona realiza sus actividades cotidianas y habituales, es decir, fuera del lugar donde reside, trabaja o estudia.

En Uruguay el turismo es uno de los principales sectores socioeconómicos. Hace años se ha visualizado como un motor de cambio y transformación del entorno en que vivimos. Su rápido crecimiento, bien sea de su lugar en el mercado o de las oportunidades de negocios que se generan, ha estimulado la idea de que vaya un peldaño más allá y coopere para incentivar el desarrollo económico del país. Esto supone pensar en términos de bienestar social y ambiental, jugando de esta forma un rol fundamental en la 'economía verde'.

De acuerdo con Paul Krugman (2010), la economía verde sería la posibilidad de que personas y empresas realicen transacciones con las que puedan generar beneficios comunes y mutuos. Se debe considerar en una amplia perspectiva, pues si por ejemplo hay una transacción de venta de una mercancía que reporta beneficios para las dos partes, pero su producción lleva 'efectos externos negativos', como costos ocultos para el medioambiente y la cultura, la economía de mercado queda totalmente deslindada. Por lo que la economía verde intenta dar respuestas a estas interrogantes, y puede tomar dos alternativas: por un lado, el dictado de normas que prohíban estas acciones y comportamientos $y$, por el otro, establecer costos elevados a quienes la lleven a cabo. 
Uruguay ha incentivado el turismo alternativo o, como también se conoce, turismo sostenible; esto ha llegado a ser un pilar fundamental dentro de la gestión pública y ha llamado la atención en el campo académico, ya que se mueve en un contexto moderno y vanguardista, en el cual participan diferentes actores públicos y privados. También se ha planteado la necesidad de reflexionar colectivamente, para ver al sector turístico con otros ojos, adoptando una visión estratégica del mismo en el plano de la construcción participativa; por tal motivo, será necesario visualizar su continuidad y relevancia para las generaciones futuras, con apoyo en los valores y la cultura que atesoran e identifican a Uruguay.

Ser sustentable ${ }^{1}$ actualmente es un valor agregado, brindado por los servicios que valoran alternativas que van más allá del tradicional 'sol y playa' y que buscan una diferenciación social (Kotler et al., 2011, pp. 660-661). En Uruguay se materializa por medio de diferentes aspectos: primero, por políticas de Responsabilidad Social Empresarial y Ambiental y, en segundo lugar, por la venta de un paquete o producto turístico completo que incluye estadías, transporte, seguridad y naturaleza. Para ello se participa activamente con la elaboración de un Plan de Turismo Sostenible, que se realizó por última vez en 2009 y alcanza hasta el 2020 (MINTUR, 2009, p. 7-10).

Utilizar la metáfora de "mundialización del turismo" significa hablar de aquellos países en pleno desarrollo que en estas turbulencias han visto y sabido aprovechar una nueva oportunidad de negocio, adaptándose a estas transformaciones y teniendo conductas sustentables con un perfil de ecoturismo. Uruguay no está alejado de esta realidad y paulatinamente está adoptando diferentes planes, para poder seguir la línea de estos cambios en los modelos turísticos, como también en la motivación y el comportamiento del turista estándar.

Según Gómez (1997, pp. 15-45), en el desarrollo organizacional se visualizan tres objetivos básicos:

1 Nota del Editor: En esta revista se diferencia la expresión sustentable de su parónima sostenible, a partir de concebir la sustentabilidad dentro de la (in) capacidad del planeta para producir y reproducir la base de bienes naturales necesarios para satisfacer las necesidades vitales de las especies bióticas y abióticas que lo habitan. Esta posición ecocéntrica se alinea con la perspectiva latinoamericana de la ecología política, las teorías decoloniales y las Epistemologías del Sur, y se desliga de la corriente desarrollista que se alterna en el uso de la expresión sustentable en este artículo. No obstante, por respeto a la autora y a la decisión de los árbitros evaluadores, mantenemos la alternancia sostenible-sustentable en el texto aprobado. 
- Tener una distribución más equitativa del ingreso entre los diferentes participantes de la sociedad.

Avanzar en el mantenimiento de la flora y fauna, mejorando el hábitat natural por medio de la creación de equipamientos e infraestructura.

Descongestionar las zonas turísticas sobresaturadas.

\section{Turismo Sostenible}

Cuando se habla de turismo sostenible, se aborda una relación indisoluble de la sociedad y el ecosistema, a partir de la comprensión del equilibrio natural de un modo armonioso que es alterado por la mano del ser humano, pero de una forma que acompasa los intereses propios de la naturaleza. En el Informe Brundtland de 1987, la Comisión Mundial sobre Medio Ambiente y Desarrollo definió el concepto de desarrollo sostenible como el "desarrollo que satisface las necesidades del presente sin comprometer la capacidad de las generaciones futuras para satisfacer sus necesidades propias". La OMT (2004) también define lo que se entiende por turismo sostenible:

Las directrices para el desarrollo sostenible del turismo y las prácticas de gestión sostenible son aplicables a todas las formas de turismo en todos los tipos de destinos, incluidos el turismo de masas y los diversos segmentos turísticos. Los principios de sostenibilidad se refieren a los aspectos ambiental, económico y sociocultural del desarrollo turístico, habiéndose de establecer un equilibrio adecuado entre esas tres dimensiones para garantizar su sostenibilidad a largo plazo.

El turismo sostenible tiene características relacionadas con la diversidad, el paisaje, la cultura, las comunidades locales y la economía rural (Lane, 1994, p. 13). Capece (1997, p. 65) lo define como una actividad que presta servicios y produce bienes para los turistas, que son aquellos individuos que se desplazan de su lugar de residencia habitual, para poder recrearse en un lugar externo a este. Estas actividades se deben planificar considerando el entorno donde se desarrollan y a las comunidades que son parte del lugar donde se presta el servicio. De las definiciones se desprende que la sostenibilidad se puede visualizar desde cuatro perspectivas diferentes (ver Figura 1):

La más conocida y popular es la sostenibilidad económica, que es la que asegura la rentabilidad y viabilidad del negocio a largo plazo. Obviamente, si los ingresos de la empresa son menores que sus egresos, no se puede pensar en una propuesta y establecimiento turístico.

La sostenibilidad social supone estar en continuo contacto con la sociedad en la que se ubica la empresa, mejorando las capacidades de la comunidad para poder recibir a los turistas. 
Siguiendo esta línea se debe analizar también la sostenibilidad ecológica, que significa que la actividad no provoque cambios nocivos e irreversibles en los diferentes ecosistemas.

Mientras que la sostenibilidad cultural implica estar en relación constante con la cultura, que es distintiva del país sin presentar grandes transformaciones en esta.

De acuerdo con Michael Porter (2009):

El principio de sostenibilidad apela al propio interés inteligente, recurriendo a menudo al llamado triple básico de resultados económicos, sociales y medioambientales. En otras palabras, las empresas deben operar de modo que aseguren el rendimiento económico a largo plazo evitando la conducta del corto plazo que es socialmente perjudicial y medioambientalmente antieconómica. Este principio funciona mejor para los asuntos que coinciden con los intereses económicos o reguladores de una empresa. (pp. 556-557)

Figura 1. Desarrollo sostenible

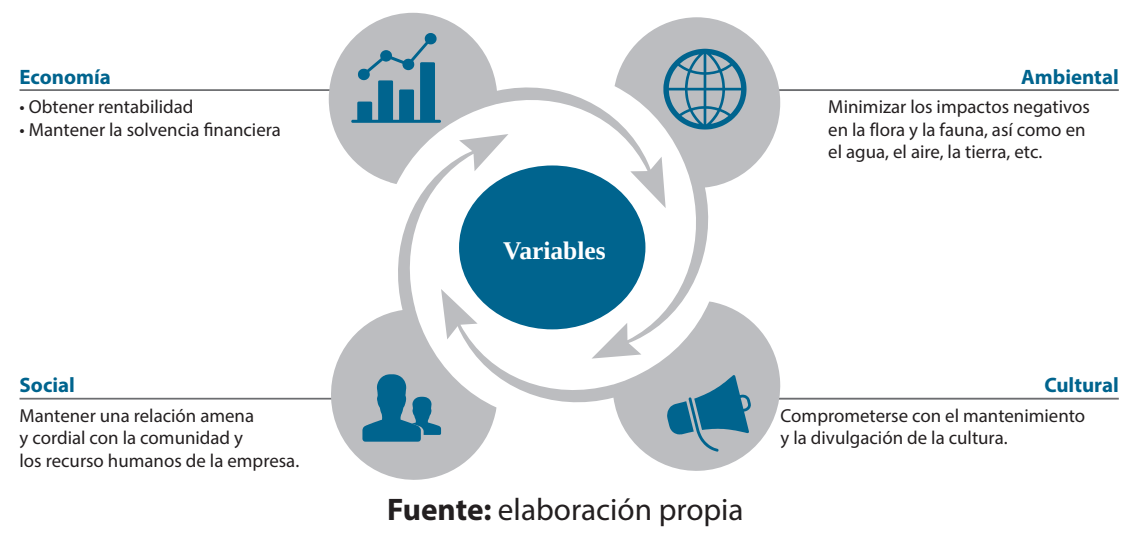

Por tanto, se puede decir que el 'binomio entre turismo y ecosistema' supone que los atentados que se lleven a cabo sobre la naturaleza no tengan una duración e intensidad tan elevada que llegue a modificar las relaciones que conforman el equilibrio natural y, en respuesta a ello, este se pueda preservar y recomponer. En contracara, si la destrucción es tan fuerte que causa un daño irreversible a las condiciones originales, se está destruyendo también el binomio que fue definido con anterioridad.

De acuerdo con Alicia Gemelli (2008, pp. 14-16), podemos decir que las acciones que realizan los turistas cuando se desplazan de sus residencias van a generar una serie de repercusiones en el destino, tanto positivas como negativas. Las positivas se visualizan en la economía y la sociedad, y paradójicamente también 
generan efectos negativos en el ambiente, a pesar de que los cambios de este último puedan perjudicar a los destinos turísticos. Por ejemplo, los grandes festivales o las fiestas de verano que se realizan en la playa sin tener en cuenta los depósitos de residuos y destruyendo el entorno, o sin tener en cuenta la preservación de las dunas y el ecosistema, son una problemática a atacar desde diferentes organismos públicos y privados. En pos de minimizar este riesgo e impacto sería recomendable buscar la forma de recolectar los residuos o, mejor aún, evitar depositarlos en el suelo; en esta segunda opción, que es la ideal, se podrían implementar prácticas de reciclaje de los residuos separando el metal, el papel, el plástico y los orgánicos, de modo que puedan reutilizarse o implementar estrategias de gestión de los mismos.

Asimismo, las dunas se ven afectadas, ya sea por el paso de la multitud que asiste o por los vehículos que estacionan sobre estas, lo que destroza la vegetación y cambia el ecosistema que impide el desmoronamiento de las mismas; por lo que sería bueno implementar normas para dichos eventos, tales como lugares predeterminados, horarios, cantidad de asistentes, decibeles de sonido, estacionamiento, etc., de modo que no afecten a los vecinos y al ecosistema. Debe tenerse en cuenta que el cambio climático — que afecta a todo el mundo - trae aparejadas tormentas que golpean sobre toda la costa, cada vez con más fuerza y frecuencia, lo que traería como consecuencia que, al perder las dunas costeras por no protegerlas debidamente, las tormentas descargarían sus furias sobre las edificaciones de toda la costa, provocando gravísimos daños a las construcciones y a la infraestructura municipal, además de la pérdida de vidas humanas (Macchi, 2017).

La idea es invertir unidades monetarias hoy en el cuidado y la preservación del medio ambiente, para que los beneficios por las ventas en un futuro sean mayores a la inversión primordial y se mantenga una relación estrecha y fructífera con la comunidad bajo el lema de 'gana-gana' para ambas partes, mejorando las ventajas competitivas organizacionales y locales (Porter, 2009, p. 219).

Se notan dos formas diferentes de pensar; por un lado, la rentabilidad económica de las organizaciones turísticas y, por el otro, la transformación social y ambiental del ecosistema (OMT, 1995). En la primera solamente se piensa en el crecimiento económico que va a ser a corto plazo, mientras que en la segunda'se abre el abanico' incluyendo una serie de aspectos relevantes que conforman el desarrollo económico, y estos aspectos son los que van ligados a la permanencia y la sostenibilidad en el tiempo. Pero, más allá de pensar en la sostenibilidad, no hay que olvidar que es el cliente quien va a pagar por la estadía en el establecimiento y a es quien la empresa deberá captar, retener y fidelizar, para lo cual debe contar con una planificación enfocada en el cliente (Amat y Campas, 2011). 
Siguiendo con las definiciones podemos adoptar una propia de la OMT (2004) en la que especifica la relevancia de esta noción:

El turismo que tiene plenamente en cuenta las repercusiones actuales y futuras, económicas, sociales y medioambientales para satisfacer las necesidades de los visitantes, de la industria, del entorno y de las comunidades anfitrionas [...] El turismo sostenible debe reportar también un alto grado de satisfacción a los turistas y representar para ellos una experiencia significativa, que los haga más conscientes de los problemas de la sostenibilidad y fomente en ellos unas prácticas turísticas sostenibles.

\section{Perspectiva del cliente}

La conducta del individuo por los cambios económicos y sociodemográficos ha variado, ya que al día de hoy posee más tiempo para viajar, las vacaciones se han vuelto más intensas, breves y frecuentes, y el modelo del turismo internacional y nacional lo ha notado (Barcos, 2009, pp. 58-60). A la hora de realizar las elecciones de sus vacaciones, el individuo va más allá de la elección de un establecimiento hotelero o de alojamiento, no lo hace solo por la categoría que presenta o por las instalaciones, sino que busca algo más que se puede visualizar en las relaciones interpersonales, con la comunidad, el ambiente, la ambientación, la atención personalizada, el lugar, el transporte, la ciudad, los comercios aledaños, etc.

De acuerdo con la teoría de March y Simon (1994), las decisiones que toman los seres humanos por su misma esencia son satisfactorias. Esto es a raíz de la motivación que se produce por medio de un estímulo-respuesta y, al haber una señal, esta misma va a provocar un sinfín de respuestas de diversa magnitud, incluso puede llegar a no producir ninguna respuesta. En palabras de los propios autores:

Los estudios empíricos de las motivaciones individuales para producir tienden a identificar: a) factores que se refieren a los objetivos de los individuos, b) factores ligados a las previsiones de las consecuencias, c) factores que se refieren al conjunto de las opciones, tal como es percibido en el momento de la decisión. (March y Simon, 1994 citados por Arocena, 2010, p. 52)

Está motivación va a surgir de un conjunto de alternativas de elección entre varias propuestas turísticas, entre las que puede escoger la mejor opción por el alojamiento, la gastronomía, las atracciones, la cultura que presenta, los paseos, el lugar de ubicación, el precio del mismo, las fechas, etc. Todas estas van a tener consecuencias, ya sean positivas o negativas, y a la vez hay que considerar que todas esas opciones y consecuencias no van a ser tales, debido a que no se consideran todas las alternativas debido a recursos, sean temporales, humanos, de información, materiales u otros. 
Aquí aplica el postulado de que la racionalidad del ser humano es limitada, por lo que va a elegir el punto medio entre lo satisfactorio y lo óptimo. Como lo plantean March y Simon, "buscar lo óptimo implica procesos infinitamente más complejos que buscar la satisfacción". (1994, p. 138). Ningún turista va a conocer de antemano todas las alternativas posibles, las que surgen de estas, los acontecimientos que pueden presentarse en un futuro o los problemas propios de la jerarquización de alternativas que realiza y sus consecuencias. Por tanto, debe simplificar la situación real para poder decidir e inclinarse por una de las alternativas que planteó.

De acuerdo con lo que alegan estos autores, "lo que una persona desea y quiere influye en lo que percibe y lo que percibe influye en lo que desea y quiere" (March y Simon, 1994, p. 148); por lo que lo cognitivo es influido por lo volitivo y viceversa, lo que a la vez supone que cualquier decisión va a ser permeable a los pensamientos del individuo y esto conduce a que sea sumamente subjetiva. Por lo tanto, y con base en lo que se entiende por desarrollo sostenible, supone que a la hora de tomar cualquier decisión satisfactoria se deben tener en cuenta las consecuencias de la misma, en términos económicos, ambientales y sociales. Por lo anterior, a la hora de decantarse y hacer una elección, el individuo debería prevenir los efectos negativos que se suponen de la misma, aunque a priori no se conozcan todos, pero sí se puede hacer una idea de la duración y la magnitud de las diferentes propuestas que presenta.

Además de brindar un buen servicio para generar relaciones duraderas con los clientes, se debe fijar un precio coherente con la calidad del servicio y las instalaciones $y$, junto con las formas de cuidado del entorno y el ambiente, informarlas a los clientes por medio de diferentes canales de comunicación y publicidad. La experiencia de la calidad del turismo toma en cuenta factores endógenos - propios del lugar donde está inserto-y exógenos — por fuera del mismo-. No obstante, hay que tener en cuenta que el turista va a evaluar la experiencia como un todo desde el transporte y las carreteras, hasta la gastronomía el comercio, la hotelería y el servicio de bar; por ende, todos los elementos que componen el producto turístico deben ser de excelentes, promoviendo continuamente la mejora de calidad en los procesos y en el resultado final.

Hay que tomar en cuenta también que el aumento del turismo no solo implica la construcción de más infraestructura hotelera o gastronómica, sino que se requiere más transporte, agua, electricidad, eliminación de residuos y desechos sólidos, servicios públicos, entre otros. Incluso, si se evalúa el caudal de turistas, se notan los cambios en la forma de ver y utilizar los diferentes recursos naturales, llegando incluso a sobrexplotarlos. Para satisfacer esta demanda hay que planificar y coordinar, ya que el exceso de la misma conlleva 
efectos negativos para los turistas, tanto como para la comunidad y el entorno en que opera. Por tanto, es vital incorporar en los preceptos de calidad la sensibilidad por la cultura y la naturaleza, así como la protección de los residentes, conjugando aspectos conservacionistas y turísticos y permitiendo un planeamiento ordenado y respetuoso de la naturaleza, siendo garantía en la conservación del medio ambiente. Esto exige que participen todos los miembros y agentes que se relacionan en el sector y la actividad.

\section{La comunicación con el cliente}

Se debe planificar una estrategia de comunicación y, a la vez, programarla para poder implementarla, con el afán de que las relaciones sean rentables. Para ello se ha de tener un diagnóstico de la situación comunicacional de la empresa, visualizando los canales formales que presenta y el modo en que se desempeñan los informales (Allen, 2009, p. 2).

Los canales formales son aquellos que poseen una estructura planificada, es decir, que los mismos son organizados de acuerdo con niveles, divisiones y departamentos. En estos, las responsabilidades son específicas y a la vez los trabajos han de estar determinados. Sin embargo, en palabras de Kreps (1995):

Los formales rara vez llegan a satisfacer las necesidades de los individuos por lo que se requiere del rumor para recolectar los tipos de información relevantes que no puede acceder por los medios formales. Cuanto menos se utilicen los medios formales, más rumores se van a generar, y más poder tienen. (p. 225)

Esto supone que los canales formales van a seguir la jerarquía que prescribe la organización, ya sea por medio de comunicación ascendente, o descendente y a la vez horizontal. Mientras que la vía clandestina también se desarrolla entre los miembros de la organización, pero en este caso tiene una estructura social no jerárquica. Se dan por un sinfín de aspectos tales como la atracción interpersonal, la interacción social y por el apetito insaciable que tienen las personas por el significado. El que posea la información va a ganar y ejercer poder dentro de la organización, por eso es que se les llama a estos miembros "líderes informales". Como lo plantea Kreps (1995):

Los canales de comunicación informal van a proporcionar información a los miembros sobre el funcionamiento de la misma, lo que puede ayudarlos a comprender la vida de la misma y dirigir de forma estratégica las propias actividades. Generalmente los formales no les proporcionan a los miembros la información que necesitan para satisfacer su curiosidad. Para crear significados efectivos sobre la vida de organización los miembros necesitan información que sea confiable, relevante y de profundidad. (p. 230) 
La comunicación con el cliente puede establecerse por diferentes medios, bien sea herramientas de publicidad, relaciones públicas, propaganda en medios masivos de comunicación, marketing directo con acciones que puede emprender el establecimiento, o ventas personalizadas directamente con el cliente o intermediarios. Para ello, de acuerdo con los postulados de Brandolini y González (2009, pp. 37-72), se deben realizar cinco instancias para conocer cómo se está comportando la organización en términos de comunicación de sus prácticas sostenibles y calidad del servicio. Tales instancias son: a) prediagnóstico: primer acercamiento a la empresa, primer contacto o referente; b) diagnóstico: devela las falencias o aciertos de la comunicación; c) planificación: realización del plan estratégico de comunicación interna teniendo en cuenta los resultados de la auditoría; en esta se han de definir los objetivos y el plan a seguir; d) ejecución del plan: se ponen en marcha las acciones contempladas y e) seguimiento: se efectúan diferentes mediciones para ver cómo evolucionan las acciones implementados. Se va corrigiendo el plan de acuerdo con la realidad que presenta la organización.

Se realiza bajo la premisa de obtener una respuesta a la latente necesidad de información sobre qué es lo que requieren los consumidores y la comunidad y, a la vez, por la llegada masiva que tienen los diferentes canales de comunicación a los potenciales consumidores explicando estas prácticas. Los clientes se pueden comunicar entre sí e intercambiar información de las diferentes experiencias, pudiendo llegar incluso a diseñar sus propios mensajes. En la mente de los clientes, las diferentes fuentes de mensajes de una cadena de establecimientos turísticos se vuelven un único mensaje. Si los mismos se contradicen, aunque sea un ápice, puede provocar confusión en la mente de los mismos y en las relaciones con ellos. Y, sí esos problemas surgen asiduamente, conlleva a que se realice una serie de mensajes que difieren entre sí para un mismo segmento de consumidores, gastando recursos y a la vez provocando una respuesta que no es la esperada.

Por lo anterior, hay que tener un control sobre la departamentalización de la empresa, debido a que diferentes secciones realizan sus mensajes y comunicaciones al consumidor. De ahí la relevancia que tiene realizar un diagnóstico y planificar la comunicación formal de la organización, ya que será en este aspecto en el que puede hacer correcciones mejorando la calidad del servicio que percibe el cliente; para ello, hay que ver a qué audiencia se va a emitir el mensaje, debe ser de una forma creíble y clara que incluya todas las prácticas, ya que en ese caso son más persuasivos. Se debe tener en cuenta la confianza, el carisma y la experiencia con que se envíe el mensaje (Amat y Campas, 2011, pp. 20-22).

En la experiencia se ve el grado de conocimiento que tiene el comunicador a la hora de emitirlo, la confianza se ve relacionada con la objetividad con que 
se transmite el mensaje y la honestidad que brinda la fuente, mientras que el carisma es la atracción que tiene el mensaje para la audiencia. Los mensajes tienen que tener en cuenta tres asuntos:

Primero, debe tener un significado esperado por la empresa, o sea, se deben enfatizar las cualidades del servicio y porqué el cliente debería adquirirlo, cómo está en concordancia con los requerimientos ambientales, las cualidades de las instalaciones, y si los recursos humanos están satisfechos, motivados y comprometidos con la estrategia, etc.

Segundo, debe haber un diferenciador, por lo que debe mostrar en qué se diferencia el servicio de otros similares y en cuáles aspectos es mejor. En esto es clave construir y acoger nuevos paradigmas, incorporando los avances teóricos y prácticos que sean razonables y que generen incentivos que contribuyan al cuidado y la preservación de la naturaleza, tratando de concientizar a los turistas y la comunidad de la relevancia en estos aspectos. También es importante certificarse por medio de diferentes normas de calidad y sustentabilidad.

Finalmente, la información debe ser creíble y honesta; hay que considerar que eso no es sencillo, ya que el cliente tiende a desconfiar de la publicidad por la cantidad de anuncios que visualiza diariamente por diferentes canales. Un individuo ve diariamente más de 1.500 anuncios, que son diferentes estímulos; muchas veces no puede atenderlos todos $y$, por ende, realiza un proceso que se conoce con el nombre de'atención selectiva'. Ahí radica la relevancia en intensificar esfuerzos para atraer la atención de los consumidores y comunicar con potencia lo que se quiere decir discerniendo la relevancia del mensaje.

Las personas también hacen una distorsión selectiva que consiste en convertir la información que ven en los anuncios, en algo que tenga un determinado significado personal, confirmando sus ideas previas sobre el servicio en cuestión; esta es la razón por la cual no descodifican los anuncios como deberían y esa descodificación puede variar entre distintos clientes.

\section{Cinco desajustes de calidad del servicio desde la perspectiva del cliente}

\section{Expectativas que tiene el cliente frente a la percepción que tiene la empresa}

Muchas veces los encargados de los establecimientos turísticos fallan a la hora de determinar qué es lo que requiere y desea el cliente, entonces elaboran un servicio que no llega a los patrones de calidad que espera el público objetivo. Más allá de hacer estudios para saber qué quiere el cliente, las empresas han de ser conscientes de que las necesidades son cambiantes, por lo que continuamente 
hay que estar adaptando el producto o servicio, de ahí la relevancia de implementar canales de comunicación ascendentes y descendentes. Esto se puede visualizar claramente en los cambios en los patrones de conducta y consumo de los potenciales clientes. La valoración a los recursos naturales se debe tener en cuenta en un contexto estratégico que alcance una visión de 'gana-gana' para todos los actores; incluso muchos establecimientos se están certificando en calidad por medio de numerosas auditorías.

En términos económicos, el usuario de los recursos naturales tenderá a no tratarlo como un bien gratuito; esto debido a que su objetivo será el mantenimiento del flujo de beneficios provenientes de los bienes y servicios proveídos por ellos. En otras palabras, el usuario racional de estos recursos tenderá a prevenir la depreciación innecesaria del patrimonio internalizado en la contabilidad empresarial y nacional. (De Tietemberg citado por Vega y Rajovitzky, 2014, p. 11).

Por ejemplo, el esquema 'sol y playa' está cambiando (si bien sigue siendo el principal destino turístico), pues ahora se buscan otro tipo de propuestas que involucren de una forma más amplia al ambiente y el ecosistema en su conjunto, tales como el turismo rural que se materializa por medio de estancias turísticas, en las que el huésped es parte de la producción y del ciclo del servicio al participar activamente en cada una de las etapas y complementándose con el lugar, con una atención cercana y sincera, una interacción con la flora y la fauna, la degustación de productos que son producidos de forma artesanal, desayunos caseros y también platos típicos de la gastronomía de Uruguay. Incluso en muchas ocasiones se practican excursiones de pesca y caza, como también cabalgatas (Turismo rural, 2013).

2. Lo que piensa la dirección de la calidad del servicio y lo que realmente se brinda

Esto se visualiza cuando se sabe qué quiere el cliente, pero la empresa no desarrolla sistemas que sean capaces de satisfacer esas expectativas, ya sea por no tener bien estandarizados los objetivos, o las tareas no están bien asignadas y delimitadas, o no parece viable, o cuando no hay compromiso. Por ejemplo, en establecimientos hoteleros de menor envergadura, en el presupuesto global no se toma en cuenta el gasto que conlleva realizar un recambio de toallas; al no considerar estos gastos, puede que no se cuente con capital suficiente para realizarlo, lo que puede perjudicar la percepción del cliente frente al establecimiento. Los directores de la empresa saben que la cantidad de toallas es escasa o no están acordes a la calidad que deberían tener, pero no invierten capital para comprar nuevas (Hetter, 2013). 


\section{Comunicación interna y gestión de los recursos humanos}

Muchas veces se saltan estos desajustes, es decir, los directivos saben qué quiere el cliente, cómo brindar esa calidad esperada, pero el personal no es capaz, está desbordado de trabajo o no quiere realizar el servicio. Los trabajadores son quienes interactúan directamente con el cliente en el momento de la verdad, lo que puede llevar a un mal comentario y a una mala impresión del cliente. Las máquinas en cambio no son tan proclives a cometer estos errores, y los clientes a la vez esperan menos de ellas, este desajuste se puede resolver implementando una mejor gestión de los recursos humanos. Por ejemplo, un cliente que realiza el registro o reserva una mesa por medios tecnológicos no espera una bienvenida muy efusiva; pero, si lo hace con el personal del establecimiento, sí lo espera. Por ello, la relevancia de capacitar al personal en atención al cliente, liderazgo, ventas.

\section{Comunicación externa}

El desajuste ocurre cuando la empresa promete más de lo que realmente puede brindar. Los directivos se deben preocupar por cumplir lo que le prometieron al cliente a la hora de arrendar la estadía. Hay que prestar especial atención a este aspecto, debido a que la palabra del establecimiento se ve mermada si le falta algo de lo que le prometieron cuando realizó la reserva; el cliente podría llevarse una mala impresión de toda la estadía generando comentarios negativos que van a repercutir en los recursos intangibles. Por ejemplo, al ofrecer una piscina climatizada que, cuando el cliente llega al hotel, está en mantenimiento y la empresa no haya tomado medidas para subsanar el problema, ni se lo haya comunicado al cliente.

\section{Diferencia de la calidad esperada y recibida}

Cuando el cliente recibe menos de lo que esperaba, al llegar se va a producir este desajuste que va a provocar insatisfacción. Es el desajuste más difícil de gestionar, ya que las percepciones son propias de cada persona, y muchas veces no se puede hacer nada para cambiarlas. Por medio del estudio de los cinco desajustes se puede incrementar la calidad del servicio prestado (ver Figura 2), debido a que se pueden ver potenciales problemas y buscar la manera de que no ocurran y, a la vez, es de ayuda para disminuir la percepción negativa del cliente sobre los mismos.

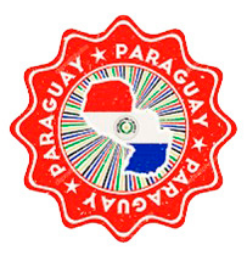


Figura 2. Cinco desajustes de la calidad

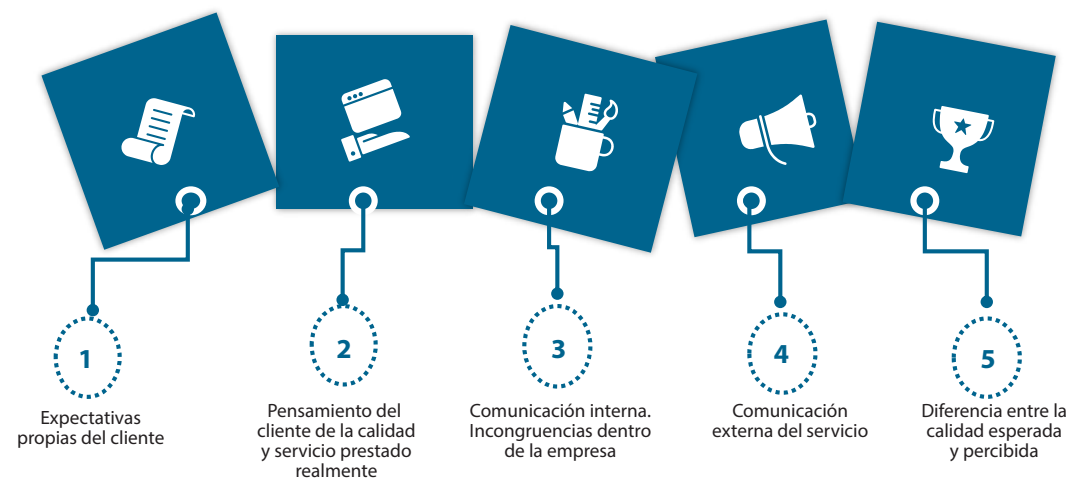

Fuente: elaboración propia

\section{Importancia de fidelizar a los clientes}

Hay que marcar una diferencia entre la retención y la fidelización del cliente. Cuando se retiene al cliente puede ser por medio de precios más bajos que la competencia o haciendo bonificaciones y descuentos; pero estos son mecanismos temporales. Mientras que la fidelización se ve en un conjunto de aspectos como lo son: el cuidado del cliente, la forma en que interactúa la compañía con el cliente, el servicio, la imagen que se lleva el cliente durante el proceso y, posteriormente, el servicio postventa en la atención de las quejas, ya que esta última es una señal de estar en contacto y en alerta. Por tanto, la imagen no será un hecho aislado, ni mucho menos una moda, sino que se deberá visualizar como un 'instrumento estratégico' (Costa, 2009, pp. 60-61).

De acuerdo con Escudero (2011), "el 68\% de los clientes se pierden por la indiferencia en la atención como en la mala atención del personal a la hora de prestar el servicio" (p. 206). Esto requiere gran competencia, flexibilidad y adaptación a los cambios de la industria y a los procesos. Cuando se dice atraer y mantener a los clientes, se deben satisfacer sus necesidades superando con creces sus expectativas. No supone solamente que vuelvan, sino que realicen comentarios positivos para otros potenciales huéspedes; cuando esto se logra, los clientes van a pagar un precio justo por el servicio que se les está brindando y en ese valor va a estar implícito un beneficio para la empresa. Esta situación es ideal para las dos partes, ya que el cliente se convierte en "amigo y prescriptor de la empresa" (Apaolaza et al., citados por Figueroa, 2011).

De acuerdo con estudios realizados por Kotler et al. (2011, pp. 10-20), para una empresa, el costo de retener a un cliente fiel es solamente el $20 \%$ del costo de atraer a un nuevo cliente. Mientras que aumentar un $5 \%$ las tasas 
de fidelidad para la empresa van a repercutir en un aumento de un $25 \%$ a un $125 \%$ del beneficio global de la empresa. La filosofía de las grandes empresas de servicios se centra en la satisfacción de las necesidades de sus clientes con el afán de incrementar su fidelidad y lealtad. A la hora de pensar en un plan de fidelización se deben cubrir las siguientes etapas:

1) Se tiene que construir la estructura de la relación, para lo que es necesario dilucidar cuáles son los clientes rentables para la empresa y aquellos potenciales que se pueden captar, por medio de una base de datos que individualice a los clientes con información de calidad.

2) Se comienza con la relación, por medio de un programa que incite a la prueba y recompensa del producto, generando preferencia con los clientes y brindándoles diferentes beneficios. Se deberá desarrollar un diálogo y una comunicación con el cliente que refuerce la interacción con ellos.

3) Por medio de una correcta segmentación, se deben realizar programas para incrementar el valor de la relación, de modo que este considere tanto el desarrollo como el costo de oportunidad, en caso de que el cliente no sea fiel a la empresa.

4) Premiar esa fidelidad con diferentes incentivos que se adecuen a las necesidades de los clientes $y$, a la vez, incrementen las ventas del establecimiento, o sea que deben ser medidas gana-gana. Asimismo, incrementando la variedad de servicios de forma periódica para mantener el interés que tiene el cliente, manteniendo la relación y midiendo la satisfacción y los errores que se puedan producir, como también el avance del ciclo de vida del cliente.

\section{Consideraciones finales}

Toda propuesta turística ha de ser razonada bajo una visión sustentable que adopte criterios éticos y económicos, y que se condicione y promocione bajo esta retórica, potenciando así el desarrollo económico del país y la región y aprovechando con ello los recursos naturales, sociales y culturales del entorno; pero, manteniendo un férreo respeto por todos estos recursos, desde la consideración de que son carácter limitado, dando garantía de desarrollo en la actualidad y para generaciones futuras. Ello exige también respeto por la identidad de la propia organización, definida como "la autorrepresentación de la organización, es decir, el discurso que asume la institución para presentarse frente a los públicos". (Sanz de la Tajada, citado por Amado, 2008, p. 65). 
Si bien es fundamental que los diferentes establecimientos vayan adquiriendo las prácticas sustentables, es necesario comunicarlas para que sean conocidas y que efectivamente tengan efectos, ya que son resultado de la planificación estratégica; por ello, es de mucha relevancia no soslayar este aspecto ya que el sector turístico tiene asignado un 'rol de cambio' que va en concordancia con el desarrollo económico de la ciudad, la región e incluso el país.

\section{Conclusiones}

A la hora de realizar una estrategia, es fundamental tener en cuenta prácticas vanguardistas que cooperen con el desarrollo y la preservación del ambiente; estas son un eslabón en la cadena de valor de cualquier establecimiento que se enfoque en el turismo. Ello requiere incorporar cuatro pilares:

La importancia de planificar el ciclo de servicio del establecimiento hotelero o gastronómico, teniendo en cuenta cada una de las etapas en las cuales se tendrá contacto con el cliente.

Dar un uso óptimo a los recursos medioambientales y sociales que presenta el lugar donde se desempeñan, pues estos van a ser parte primordial a lo largo del ciclo del servicio.

Tener un cuidado óptimo en cada detalle o pasos que componen las etapas del servicio, ya que son ellos los que harán una experiencia única e inolvidable. Para ello, es fundamental contar con canales de comunicación formales internos y externos que sean completos y se ajusten a las necesidades de información de la organización y de sus clientes, para poder comunicar las propuestas de valor y las ventajas competitivas.

Adoptar una cultura de aprendizaje continuo, aprovechando las reacciones y los comentarios que revelan los sentimientos y las percepciones de los clientes; para ello, habría que tener en cuenta diferentes indicadores e implementar las sugerencias para mejorar la calidad del servicio prestado y así lograr fidelizar al cliente.

La cadena de valor se va a romper en el eslabón más débil, por lo que es importante considerarlo. Ser socialmente responsable no se construye en un sólo día, sino que es el resultado de la suma de diferentes acciones y decisiones que se van forjando en el día a día a lo largo del tiempo; por el contrario, los aspectos importantes sí se destruyen en un día con una mala acción o decisión. Por eso, la relevancia de planificar y gestionar todas las acciones, así como comunicarlas adecuadamente por los medios de comunicación que adopte la organización. 
Asimismo, estos modelos a tener en cuenta contribuyen al logro de objetivos de los establecimientos turísticos, desde varios puntos de vista: en primer lugar, por la imagen externa que se ve beneficiada a causa de la comunicación, gracias a las respuestas y retroalimentaciones de los clientes y la comunidad, además de los preceptos éticos. En segundo lugar, hay que prestarle especial atención a los potenciales conflictos de intereses que tienen los clientes, aplicando la matriz de los cinco desajustes de la calidad, para poder visualizar si el servicio prestado se asemeja al comunicado y, en caso de no ser así, tomar medidas a tiempo.

$* * *$

\section{Referencias bibliográficas}

1. Allen, R. (2009). Creating an internal communications strategy from scratch where do you start? Melcrum Publishing.

2. Amado, A. (2008). Auditoría de comunicación: un método de análisis de las comunicaciones públicas. La Crujía ediciones.

3. Amat, O. y Campas, F. (2011). Contabilidad, control de gestión y finanzas de hoteles: con casos prácticos resueltos. Profit.

4. Arocena, J. (2010). Las Organizaciones Humanas. De la racionalidad mecánica a la inteligencia organizacional. Grupo Magro editores.

5. Barcos, S. (2009). Una aproximación teórica práctica al estudio de las organizaciones turísticas y su administración. Ediciones Haber

6. Brandolini, A. y González, M. (2009). Comunicación Interna. La Crujía.

7. Capece, G. (1997). Turismo sostenido y sustentable. Una visión holística. Agencia periodística CID.

8. Costa, J. (2009). Imagen corporativa en el Siglo XXI: la auditoría estratégica global. La Crujía.

9. Escudero, M. (2011). Gestión comercial y servicio de atención al cliente. Editorial Paraninfo.

10. Figueroa, M. (2011). Fidelización de clientes: concepto y perspectiva contable. Tec Empresarial, 5(3), 29-35.

11. Gemelli, A. (2008). Estrategia de Turismo sustentable. Programa de Naciones Unidas para el Desarrollo - PNUD.

12. Gómez, V. (1997). Planificación Económica del Turismo: de una estrategia masiva a una artesanal ( $1^{\text {a }}$ ed.). Trillas. 
13. Hetter, K. (2013, 22 de marzo). El dilema de las toallas de los hoteles: ¿cambiar o reutilizar? Expansión. http://expansion.mx/planetacnn/2013/03/22/eldilema-de-las-toallas-de-los-hoteles-cambiar-o-reutilizar

14. Kotler, P., Bowen, J., Makens, J., García, J. y Flores, J. (2011). Marketing turístico. Pearson educación.

15. Kreps, G. (1995). La comunicación en las organizaciones. Addison - Wesley Iberoamericana.

16. Krugman, P. (2010, 25 de abril). Cómo construir una economía verde. El País. https://elpais.com/diario/2010/04/25/negocio/1272201265_850215.

17. Lane, B. (1994). Sustainable rural tourism strategies: A tool for development and conservation. Journal of sustainable tourism, 2(1-2), 102-111.

18. Macchi, F. (2017, 4 de febrero). Dunas en Uruguay: una costa vulnerable. El Observador. https://www.elobservador.com.uy/dunas-uruguay-una-costavulnerable-n1025856

19. March, J. y Simon, A. (1994). Teoría de las organizaciones. Editorial Ariel.

20. Ministerio de Turismo y Deporte del Uruguay - MINTUR (2009, junio). Plan Nacional de Turismo Sostenible. Gobierno de Uruguay. http://apps.mintur. gub.uy/Plantur/components/Plan\%20Turismo\%20Sostenible_final.pdf

21. Organización Mundial del Turismo - OMT (1995). Carta de turismo sostenible. Conferencia Mundial de Turismo Sostenible. https://www.e-unwto.org/doi/ pdf/10.18111/unwtodeclarations.1995.21.15.1

22. Organización Mundial del Turismo - OMT (2004). Turismo sustentable. http:// sdt.unwto.org/es/content/definicion

23. Porter, M. (2009). Ser competitivo. Ediciones Deusto.

24. Turismo rural: alojamiento y estancias turísticas en Uruguay. (2013, 20 de septiembre). Portal de América. https://www.portaldeamerica.com/ blogdeviajes/index.php/item/290-turismo-rural-alojamiento-y-estanciasturisticas-en-uruguay

25. Vega, R. y Rajovitzky, A. (2014). Contabilidad Ambiental. Editorial Académica Española.

Para citar este artículo:
Suárez B., M. (2021). Calidad del turismo sostenible desde la perspectiva del cliente. Teuken Bidikay, 12(18), 179-196 doi: 10.33571/teuken.v12n18a9

$\mathrm{Ge}^{*}: \mathrm{AMV}$. 